\title{
ESTUDO DAS RELAÇÕES ENTRE MASTIGAÇÃO E POSTURA DE CABEÇA E PESCOÇO - REVISÃO SISTEMÁTICA
}

\author{
Study of the relationship between \\ chewing and head and neck posture - systematic review
}

\author{
Thais Myrian Aragão Mélo ${ }^{(1)}$, Celina Cordeiro de Carvalho ${ }^{(2)}$, Ada Salvetti Cavalcanti ${ }^{(3)}$, \\ Mário Genuíno Dourado Filho ${ }^{(4)}$, Paulo Fernandes Pinheiro Junior ${ }^{(5)}$, Hilton Justino da Silva ${ }^{(6)}$
}

\begin{abstract}
RESUMO
Tema: mastigação e postura de cabeça e pescoço. Objetivo: realizar uma revisão sistemática para observar se existe uma interferência recíproca entre a função da mastigação e a postura de cabeça e pescoço. Conclusão: existe reciprocidade entre a função da mastigação e alterações na região de cabeça e pescoço, apesar de existirem dúvidas quanto a qual região promove primariamente as alterações na outra. Assim, são importantes mais estudos utilizando métodos de avaliação mais direcionados e mensuráveis para cada região para fornecer dados mais acurados.
\end{abstract}

DESCRITORES: Mastigação; Postura; Cabeça; Pescoço

\section{INTRODUÇÃO}

A mastigação é considerada uma das funções mais importantes do sistema estomatognático e seu desenvolvimento e aperfeiçoamento dá-se ao longo do crescimento humano $0^{1-3}$. Para que ocorra de forma eficiente, é necessária a saúde dos dentes e a possibilidade adequada dos movimentos mandibulares, que são controlados pelas articulações temporomandibulares (ATM's) e pelo sistema

(1) Fisioterapeuta; Mestranda em Patologia pela Universidade Federal de Pernambuco.

(2) Fisioterapeuta; Docente do curso de Fisioterapia da Estácio - FIR, Recife, PE; Doutora em Neurociências pela Universidade Federal de Pernambuco.

(3) Terapeuta Ocupacional; Mestranda em Patologia pela Universidade Federal de Pernambuco.

(4) Medico Radiologista; Membro titular do Colégio Brasileiro de Radiologia - CBR, São Paulo-SP, Brasil; Mestrando em Patologia pela Universidade Federal de Pernambuco.

(5) Cirurgião dentista; Especialista em prótese dentária; Mestrando em patologia pela Universidade Federal de Pernambuco.

(6) Fonoaudiólogo; Vice-coordenador do Programa de Pósgraduação em Patologia, nível mestrado; Docente do curso de Fonoaudiologia da Universidade Federal de Pernambuco, Universidade Federal de Pernambuco - UFPE, Recife, Pernambuco, Brasil; Doutor em Nutrição pela Universidade Federal de Pernambuco.

Conflito de interesses: inexistente neuromuscular ${ }^{4}$. O desenvolvimento harmônico das estruturas envolvidas na mastigação depende da associação entre equilíbrio muscular e função, o que mostra que alterações isoladas inexistem ${ }^{5}$. Entretanto, os mecanismos mastigatórios são bastante flexíveis, adaptando-se às compensações oriundas das deficiências ou situações ocorridas ${ }^{6}$.

A influência da atividade postural dos tecidos moles, no desenvolvimento dentofacial, é considerada mais importante que os efeitos da contração muscular e os movimentos mandibulares ${ }^{7}$. Conexões neurais existem entre os sistemas sensóriomotores cervical e trigeminal ${ }^{8}$, mostrando que fortes ligações neuromusculoesqueléticas e neurofisiológicas estão envolvidas na inter-relação entre as regiões orofacial e cervical ${ }^{9}$. Isto reforça a existência de atividade simultânea e coordenada entre os músculos do pescoço e mandíbula ${ }^{8}$.

Apesar de a mastigação ser descrita em diversos estudos como tendo aspectos relacionados com a musculatura cervical, a associação ou interdependência entre esta e a postura de cabeça e pescoço ainda não foi investigada de forma sistematizada. $O$ objetivo desse estudo é realizar uma revisão sistemática para observar se existe uma interferência recíproca entre a função da mastigação e a postura de cabeça e pescoço em indivíduos normais. 


\section{MÉTODO}

Foi realizada uma revisão sistemática através de pesquisa eletrônica, entre os meses de setembro e outubro de 2010, utilizando as bases de dados da MEDLINE, LILACS e SCIELO. Para a pesquisa foram utilizados os seguintes descritores, confirmados via DeCS (Descritores em Ciências da Saúde), em português/inglês: mastigação/chewing, postura/posture, cabeça/head, pescoço/neck. Combinações foram feitas entre estes para a realização da pesquisa.

Os critérios de inclusão foram artigos originais; sem limites de período de publicação; em populações humanas adultas (> 18 anos); indivíduos estudados normais, sem distúrbios temporomandibulares (DTM) e sem disfunções neuromusculares com interferência na musculatura mastigatória e/ ou na postural, envolvidas no suporte de cabeça e pescoço; os que faziam associação direta entre a mastigação e a postura da cabeça e coluna cervical e os classificados como journal article.

Para aquisição dos artigos selecionados para esta revisão sistemática etapas foram seguidas. $\mathrm{Na}$ primeira, com a procura pelos descritores mencionados, foram encontrados 676 artigos. Após se estipular os seguintes limites para a busca de estudos: humanos, adultos (idade superior a 18 anos) e os com publicação somente nos idiomas inglês, espanhol e português, 302 artigos foram excluídos. Assim, passou-se para a leitura do título, com a qual houve a exclusão de 357 artigos, sendo dois retirados por serem repetidos. A etapa seguinte envolvia a leitura dos resumos, resultando em mais seis artigos retirados do estudo, sendo, então, selecionados 11 artigos. A etapa seguinte foi a de análise do artigo, se neles havia avaliação ou comparação direta entre mastigação e postura de cabeça e pescoço em adultos, com métodos coerentes com os critérios de inclusão. Nesta, foram excluídos seis artigos e adicionados três artigos de interesse através da observação da referência. Totalizando oito artigos selecionados para a revisão sistemática.

Para melhor apresentação dos resultados optou-se por considerar as seguintes variáveis, nos oito artigos selecionados: autor/ano, país, amostra, método de avaliação utilizado e conclusão, organizadas por ano da publicação (conforme descrito na Tabela 1).

\section{REVISÃO DA LITERATURA}

Após a análise dos 676 artigos encontrados nesta revisão foi observado carência de trabalhos que abordem a relação entre mastigação e postura de cabeça e pescoço em uma população de indivíduos normais. Apenas oito estudos abordaram essa relação de forma mais clara em seus objetivos.

Um aspecto que chama a atenção nos estudos selecionados é o período de publicação. Neste estudo, nota-se que, apesar de não ser estipulado limite de ano para a pesquisa dos artigos, os primeiros estudos foram publicados a partir da década de $1980^{10,11}$, com um espaçamento de quase uma década nas publicações para os dois novos existentes nos anos de $1990{ }^{12,13}$. Entre 2000 e 2006, houve uma intensificação na publicação de estudos que abordavam essa temática, com quatro artigos publicados, ou seja, metade das publicações desta revisão. Nos últimos cinco anos desde a última publicação não foram encontrados nesta revisão outros trabalhos, a partir dos critérios de inclusão utilizados. Essa situação pode ser decorrente do interesse maior em observar a função da mastigação correlacionando-a com alterações na postura em indivíduos com algum comprometimento temporomandibular, não sendo, então, incluídos nessa revisão. É importante a realização de pesquisas que contemplem achados em uma população normal, visto sua relevância como forma de parâmetro para o que é aceitável ou passível de ser encontrado.

Outro aspecto relevante diz respeito a pouca variação nos países de realização dos estudos, onde a maioria $(62.5 \%)^{8,10,12,14,15}$ foi desenvolvida na Suécia, sendo três destes ${ }^{8,14,15}$ produzidos por um mesmo grupo de pesquisadores/professores. Os demais estudos foram realizados nos Estados Unidos $(25 \%)^{11,13}$ e no Japão $(12,5 \%)^{16}$. Constatase, então, a ausência de trabalhos com este enfoque em países sul-americanos, o que sugere o pouco investimento acadêmico na realização de projetos que visem validar ou reforçar a idéia de interação neuromuscular entre funções, em uma população adulta saudável, nos países menos desenvolvidos.

Ainda, os estudos presentes nesta revisão são todos do tipo observacional. Em estudos observacionais não existe aleatoriedade na coleta dos voluntários participantes, o que pode, de certa forma, tendenciar os resultados. Estudos randomizados, como citado por Souza ${ }^{17}$, são mais indicados, quando possível, devido a seu aspecto não conveniente, aleatório e de maior relevância clínica, uma vez que minimizam os fatores confusionais sobre relações de causa-efeito, aumentando o poder estatístico dos achados.

Outra particularidade dos estudos é o número limitado de indivíduos avaliados, que vão de $12^{11,14-16}$ a $40^{13}$ indivíduos, e a ausência do uso do cálculo amostral em todos os trabalhos, aumentando 
Tabela 1 - Resultados dos artigos selecionados pela pesquisa eletrônica sistemática

\begin{tabular}{|c|c|c|c|c|}
\hline AUTOR/ANO & PAÍS & $\begin{array}{l}\text { TIPO DE } \\
\text { ESTUDO }\end{array}$ & AMOSTRA & MÉTODO DE AVALIAÇÃO \\
\hline $\begin{array}{l}\text { Winnberg } \\
\text { et al, } 1983\end{array}$ & Suécia & Observacional & $\begin{array}{l}\mathrm{N}=20 \\
\text { Grupo de } \\
\text { estudo }\end{array}$ & $\begin{array}{l}\text { sEMG do masseter esquerdo e } \\
\text { suprahióideos esquerdo durante } \\
\text { ciclo mastigatório com cabeça na } \\
\text { posição neutra, inclinada pra } \\
\text { frente e retroposicionada. }\end{array}$ \\
\hline $\begin{array}{l}\text { Goldstein } \\
\text { et al, } 1984\end{array}$ & $\begin{array}{l}\text { Estados } \\
\text { Unidos }\end{array}$ & Observacional & $\begin{array}{l}\mathrm{N}=12 \\
\text { Grupo de } \\
\text { Estudo }\end{array}$ & $\begin{array}{l}\text { Cinesiografia mandibular para os } \\
\text { componentes verticais e ântero- } \\
\text { posteriores da trajetória de } \\
\text { fechamento mandibular na } \\
\text { posição sentada natural, com } \\
\text { anteriorização da cabeça, com } \\
\text { anteriorização máxima de } \\
\text { cabeça e postura do pescoço } \\
\text { reta. }\end{array}$ \\
\hline $\begin{array}{l}\text { Kiliaridis } \\
\text { et al, } 1995^{12}\end{array}$ & Suécia & Observacional & $\begin{array}{l}\mathrm{N}=13 \\
\text { Grupo de } \\
\text { estudo }\end{array}$ & $\begin{array}{l}\text { Fotografias padronizadas do } \\
\text { perfil facial nas mudanças da } \\
\text { postura de cabeça. } \\
\text { Método optoeletrônico (dados } \\
\text { quantitativos tridimensionais). }\end{array}$ \\
\hline
\end{tabular}

\section{CONCLUSÃO}

Postura da cabeça afetou todos os parâmetros EMG analisados. Nenhuma reciprocidade entre masseter e suprahióideo existiu na mastigação.

Postura antero-posterior de cabeça e pescoço tem efeito imediato na trajetória do fechamento mandibular. Cabeça anteriorizada diminui a distância vertical de fechamento mandibular. Quando posteriormente, a excursão anterior da mandíbula no espaço interoclusal diminui.

Fadiga dos músculos mastigatórios não tem influência significante na média da postura cranio-vertical da cabeça, embora haja mudanças na posição postural da mandibular. Diferenças interindividuais na composição das fibras musculares mastigatórias.

\begin{tabular}{|c|c|c|c|c|}
\hline $\begin{array}{l}\text { Higbie et al, } \\
1999^{13}\end{array}$ & $\begin{array}{l}\text { Estados } \\
\text { Unidos }\end{array}$ & Observacional & $\begin{array}{l}\mathrm{N}=40 \\
\text { Grupo de } \\
\text { Estudo }\end{array}$ & $\begin{array}{l}\text { Medidas da abertura mandibular } \\
\text { vertical com a cabeça } \\
\text { anteriorizada, neutra e retraída. }\end{array}$ \\
\hline
\end{tabular}

A posição da cabeça e pescoço afeta a função temporomandibular, sendo um importante fator para aquisição da abertura mandibular vertical em adultos saudáveis.

Encontrada diferenças no padrão funcional entre sistema

\begin{tabular}{lll}
\hline Zafar et al, Suécia & Observacional & $\mathrm{N}=24$ \\
& & $\begin{array}{l}\text { Grupo de } \\
\text { estudo }\end{array}$
\end{tabular}

Sistema optoeletrônico sem fio (tridimensional) dos movimentos da mandíbula e cabeça-pescoço simultaneamente registrados. Realizada 2 medidas (rápida e lenta) de aberturas e fechamentos voluntários máximos da mandíbula.

\begin{tabular}{lll}
\hline Eriksson & Suécia & Observacional \\
et al, $\mathbf{2 0 0 0}^{14}$ & & $\begin{array}{l}\mathrm{N}=12 \\
\text { Grupo de } \\
\text { estudo }\end{array}$
\end{tabular}

Movimentos tridimensionais da mandíbula e cabeça simultaneamente registrados através do sistema optoeletrônico sem fio. Avaliados em 2 sessões consecutivas enquanto realiza 3 tarefas motoras padronizadas. de movimentos da cabeça entre posições iniciais, de pico e finais, entre as fases de abertura e fechamento mandibular e entre velocidades rápidas e lentas. Ligação funcional entre as regiões craniocervical e temporomandibular humana.

Suporta a idéia de relação neuromuscular temporomandibular e craniocervical. Sugere que os movimentos mandibulares funcionais resultam da ativação tanto da mandíbula como dos músculos do pescoço, conduzindo para movimentação simultânea nas articulações temporomandibular, atlantooccipital e coluna cervical.

\begin{tabular}{|c|c|c|c|c|}
\hline $\begin{array}{l}\text { Häggman- } \\
\text { Henrikson } \\
\text { et al, } 2004^{15}\end{array}$ & Suécia & Observacional & $\begin{array}{l}\mathrm{N}=12 \\
\text { Grupo de } \\
\text { estudo }\end{array}$ & $\begin{array}{l}\text { Movimentos tridimensionais da } \\
\text { mandíbula e cabeça } \\
\text { simultaneamente registrados } \\
\text { pelo sistema optoeletrônico sem } \\
\text { fio. Os bolus foram avaliados } \\
\text { através de } 4 \text { testes padronizados } \\
\text { de diferentes texturas e } \\
\text { quantidade de fragmentos. }\end{array}$ \\
\hline
\end{tabular}

Um tamanho maior e uma textura mais dura do alimento correlaciona-se com uma maior extensão de cabeça e maiores amplitudes de movimento mandibular e de cabeça. Sugere que o comportamento da cabeça é modulado pelos estímulos sensório-motores mandibulares de modo tarefa-dependente durante a mastigação.

\begin{tabular}{|c|c|c|c|c|}
\hline $\begin{array}{l}\text { Shimazaki } \\
\text { et al, } 2006^{16}\end{array}$ & Japão & Observacional & $\begin{array}{l}\mathrm{N}=12 \\
\text { Grupo de } \\
\text { estudo }\end{array}$ & $\begin{array}{l}\text { Atividade do músculo masseter e } \\
\text { do esternocleidomastóideo, } \\
\text { assim como o movimento } \\
\text { mandibular e de cabeça } \\
\text { examinados usando tanto a EMG } \\
\text { quanto o sistema de captura de } \\
\text { movimento. }\end{array}$ \\
\hline
\end{tabular}

Existe relação funcional entre as do músculo esternocleidomastóideo e entre os movimentos de cabeça e mandíbula durante a mastigação. atividades do músculo masseter e 
a possibilidade de os resultados não apresentarem reprodutibilidade na população em geral.

Quanto aos métodos de avaliação estudados, o mais prevalente na análise do processo mastigatório foi o sistema optoeletrônico ${ }^{8,12,14,15}$, com o qual há a captação do movimento de forma tridimensional, através de marcadores superficiais fixados na pele nas regiões a serem estudadas ${ }^{8}$. Entretanto, a falta de elementos descritivos nos métodos e a ausência do uso desse sistema em nossa realidade não oportunizaram o esclarecimento do mesmo.

Outro método de avaliação da mastigação que, apesar de ter sido citado em apenas dois estudos nesta revisão, foi bastante recorrente durante a pesquisa eletrônica é a eletromiografia de superfície (sEMG). Esta vem sendo amplamente utilizada por ser um método eficaz, não-invasivo, de rápida aplicação, com baixo custo ${ }^{18-20} \mathrm{e} \mathrm{livre} \mathrm{de} \mathrm{desconfortos} \mathrm{e}$ radiação ${ }^{20}$. Visa registrar os potenciais de ação que ocorrem com a contração muscular ${ }^{21}$, quantificando a função muscular ${ }^{18,20}$, com informações como da amplitude, freqüência e duração ${ }^{18}$, o que o torna útil no complemento diagnóstico e preventivo ${ }^{22}$ de alterações musculoesqueléticas e no acompanhamento dos efeitos terapêuticos nessa musculatura ${ }^{19,23}$. Porém, a maioria dos estudos destina-se a observar sua utilização para observar o seguimento das disfunções temporomandibulares ${ }^{22}$, não expressando a potencialidade de sua aplicação na avaliação da função muscular de outras regiões, para refletir, conseqüentemente, as estruturas que interferem para harmonia dessa função.

Para avaliação da postura de cabeça e pescoço, não foi observado nos estudos a realização de avaliação especifica. Em todos os estudos selecionados nesta revisão, a avaliação ocorreu em concomitância com a avaliação da mastigação, sem a intenção de analisá-la isoladamente. Isto pode ser reflexo dos objetivos das pesquisas que eram ou de investigar a influência de alterações solicitadas da postura de cabeça e pescoço em estruturas que interferem na mastigação $0^{10-13}$, ou de demonstrar como se comportava a posicionamento da cabeça durante um ato mastigatório ${ }^{8,14-16}$. Ou seja, a prioridade não era a de analisar se uma alteração real na postura craniocervical estaria relacionada com alterações na função mastigatória. Torna-se importante a realização de estudos que avaliem a postura cervical isoladamente e os resultados comparar com os achados da mastigação.

Existem diversos métodos que contribuem para avaliação postural sendo utilizados atualmente. Os mais prevalentes nos estudos são a goniometria (quantificando a amplitude de movimento daquela região de forma passiva e/ou ativa) e a fotogrametria (com fotografias digitalizadas da postura do indivíduo analisadas por software específico para mensuração de distâncias e ângulos) ${ }^{24,25}$, que juntos podem fornecer dados mais objetivos, completos e fidedignos do que métodos que se utilizam da estimativa visual.

No decorrer do processo de pesquisa, a forma de avaliação postural mais recorrente foi o registro fotográfico. Com a fotogrametria, como no estudo de Pasinato et al ${ }^{24}$, fotografias do indivíduo em diferentes planos são tiradas para se ter uma visão mais ampla daquela postura. Estas fotografias digitalizadas são, então, analisadas por um software que traçará, com os pontos de referências estabelecidos por um protocolo, retas, formando ângulos que mostrarão como está o alinhamento daquele seguimento corporal analisado ${ }^{24,26}$. A fotogrametria digital é uma alternativa para a avaliação quantitativa das assimetrias posturais na avaliação postural, com medidas lineares e angulares, o que o torna o método mais concordante entre examinadores diferentes, quando comparado com a avaliação postural visual ${ }^{27}$.

Alguns autores ${ }^{28,29}$ propõem, ainda, um teste avaliativo que utiliza a análise da biomecânica em terceira dimensão (3D) simulando posturas e movimentos e traçando relações entre eles através de um programa computadorizado que possui modelos padronizados já estudados, que é o modelo elementar finito (FEM). Estes merecem ser melhor estudados e aplicados para maior aperfeiçoamento e utilização.

Ao observar as conclusões dos estudos selecionados, nota-se que a postura de cabeça e pescoço influencia não somente os parâmetros eletromiográficos da musculatura mastigatória ${ }^{10}$, mas também a potencialidade de amplitude de movimento mandibular ${ }^{11,13}$, uma vez que afeta a função temporomandibular ${ }^{13}$. Aliás, como afirmado por Zaffar et $a^{8}{ }^{8}$, o movimento mandibular funcional envolve as articulações temporomandibular (ATM), atlanto-occipital e da coluna cervical. E, embora não seja o objetivo dessa revisão, sendo excluídos os artigos que estudavam a disfunção temporomandibular, foi possível observar no decorrer da pesquisa que a hipótese da relação entre as alterações na ATM e a postura de cabeça e pescoço também era reforçada nestes indivíduos.

Observa-se, então, que é possível ser estabelecida uma relação direta entre a postura de cabeça e pescoço adotada e alterações em aspectos que interferem diretamente na mastigação, como musculatura 10,14,16, trajetória e postura mandibular ${ }^{8,11-14,16}$. E, ainda, que a postura da cabeça adotada é dependente também da atividade mecânica exigida durante a mastigação ${ }^{15}$. 
Contudo, apesar de a hipótese de ligação funcional entre as regiões temporomandibulares e craniocervicais ter suporte ${ }^{8,14,16}$, há contradição quanto a qual região quando atingida por algum tipo de alteração influencia primariamente nas características da outra ${ }^{29}$. Até porque, para que a mandíbula se movimente de forma funcional, existe uma ação concomitante da mesma com a coluna cervical e a musculatura do pescoço $8,11,14,16$. De acordo com um estudo desenvolvido por Catanzariti et al ${ }^{30}$, a contração dos masseteres está associada ao aumento da atividade elétrica de músculos que parecem manter a estabilidade de cabeça e pescoço durante a oclusão - trapézio e esternocleidomastóideo, corroborando com o achado de Motoyoshi et al ${ }^{28}$, que afirma que alterações na oclusão influencia a postura de cabeça. Já, segundo Schinestsck et al ${ }^{31}$, é a posiçäo anormal da cabeça que altera as relações biomecânicas cranio-cervicais e cranio-mandibulares, interferindo no crescimento e na postura corporal do indivíduo, visto que as modificações ocorrem em cadeia.

\section{CONCLUSÃO}

Com esta revisão sistemática, foi observada uma interferência recíproca entre a função da mastigação e alterações na região de cabeça e pescoço. Entretanto, há dúvidas quanto a qual região promove primariamente as modificações ou adaptações com relação à outra, já que as compensações ocorrem em cadeia.

Embora os achados mostrem-se favoráveis à associação entre mastigação e postura de cabeça e pescoço, estes resultados podem não estar demonstrando de forma fidedigna o achado na população em geral, visto a ausência de randomização e mascaramento nos estudos. Assim, tornase importante a realização de trabalhos com a adoção de estratégias na tentativa de diminuir os viéses da pesquisa e com a utilização de métodos de avaliação direcionados e mensuráveis para cada região, fornecendo dados mais representativos, quantitativos e fidedignos, para poder refletir maior acurácia e impacto dos resultados.

\begin{abstract}
Subject: head and neck chewing and posture. Purpose: to carry out a systematic review with the purpose of observing if there is a reciprocal interference between the masticatory function and of head and neck posture. Conclusion: there is reciprocity between the chewing function and the alterations in the head and neck regions, although there are doubts about which region primarily promotes changes in the other one. Thus, it is important to carry on more studies using more targeted and measurable assessment methods for each region in order to provide more accurate data.
\end{abstract}

KEYWORDS: Chewing; Posture; Head; Neck

\section{REFERÊNCIAS}

1. Duarte LIM. Relação entre maloclusão e mastigação [Monografia]. Londrina (PR): Curso de Especialização em Fonoaudiologia Clínica (CEFAC); 2000.

2. Saconato M, Guedes ZCF. Estudo da mastigação e da deglutição em crianças e adolescentes com sequência de Möbius. Rev. Soc Bras Fonoaudiol. 2009;14(2):165-71.

3. Felício CM, Melchior MO, Silva MAMR, Celeghini RMS. Desempenho mastigatório em adultos relacionado com a desordem temporomandibular e com a oclusão. Pró-Fono. 2007; 19 (2):151-8.
4. Cattoni DM. Alterações da mastigação e deglutição. In: Ferreira LP, Befi-Lopes DM, Limongi SCO, organizadores. Tratado de fonoaudiologia. São Paulo: Roca; 2004: 277-91.

5. Munõz GC, Carvalho CS, Misaki JK, Gomes ICD, Carvalho ARR. Análise dos potenciais elétricos do músculo masseter durante a mastigação de alimentos com rigidez variada. Rev CEFAC. Abr-jun, 2004; 6 (2): 127-34.

6. Ahlgren J. Mechanism of mastication. Acta odontol Scand. 1966; 24(Suppl 44):1-109.

7. Proffitt WR. Equilibrium theory revisited. Angle Orthod. 1978; 48:175-86.

8. Zafar, H.; Nordh, E.; Eriksson, P.-O. Temporal coordination between mandibular and head-neck 
movements during jaw opening-closing tasks in man. Archives of Oral Biology. 2000; 45:675-82.

9. De Laat A, Meuleman H, Stevens A, Verbeke G. Correlation between cervical spine and temporomandibular disorders. Clin Oral Investig. 1998; 2:54-7.

10. Winnberg A, Pancherz $H$. Head posture and masticatory muscle function. An EMG investigation. Eur J Orthod. 1983; 5(3): 209-17.

11. Goldstein DF, Kraus SL, Williams WB, Glasheen-Wray M. Influence of cervical posture on mandibular movement. J Prosthet Dent. Sep, 1984; 52(3): 421-6.

12. Kiliaridis S, Katsaros C, Karlsson S. Effect of masticatory muscle fatigue on cranio-vertical head posture and rest position of the mandible. Eur $\mathrm{J}$ Oral Sci. Jun, 1995; 103(3):127-32.

13. Higbie EJ, Seidel-Cobb D, Taylor LF, Cummings GS. Effect of head position on vertical mandibular opening. J Orthop Sports Phys Ther. Feb, 1999; 29(2):127-30.

14. Eriksson P-O, Häggman-Henrikson B, Nordh E, Zafar $\mathrm{H}$. Co-ordinated mandibular and head-neck movements during rhythmic jaw activities in man. $J$ Dent Res. 2000; 79(6):1378-84.

15. Häggman-Henrikson B, Eriksson P-O. Head movements during chewing: relation to size and texture of bolus. J Dent Res. 2004; 83(11):864-8.

16. Shimazaki K, Matsubara N, Hisano M, Soma K. Functional relationships between the masseter and sternocleidomastoid muscle activities during gum chewing: the effect of experimental muscle fatigue. Angle Orthodontist. 2006; 76(3): 452-8.

17. Souza RF. O que é um estudo clínico randomizado? / What is a randomized clinical trial? Medicina (Ribeirão Preto). 2009; 42(1): 3-8.

18. Moraes KJR, Cunha RA, Lins OG, Cunha DA, Silva HJ. Eletromiografia de Superfície: Padronização da Técnica. Neurobiologia. Jul-set, 2010; 73 (3): 151-8.

19. Pernambuco LA, Cunha RA, Lins OG; Leão JC, Silva HJ. A eletromiografia de superfície nos periódicos nacionais em fonoaudiologia. Rev. CEFAC. Jul-ago, 2010; 12(4):685-92.

http://dx.doi.org/10.1590/S1516-18462011005000125

RECEBIDO EM: 17/012/2010

ACEITO EM: 02/06/2011

Endereço para correspondência:

Thais Myrian Aragão Mélo

Rua Manoel de Carvalho, № 226 apto 802 -

Aflitos - Recife - PE

CEP: $52050-370$

E-mail: thaismaragao@ hotmail.com
20. Goldstein LB. The use of surface electromyography in objective measurement of the muscle function in facial pain / temporomandibular dysfunction patients. Funct Orthod. 2000; 17:26-9.

21. Cyrillo FN, Torriani C. Biofeedback: conceitos básicos e aplicabilidade clínica. Rev Fisioter UniFMU. 2003; 1(1): 11-8.

22. Malta J, Campolongo GD, Barros TEP, Oliveira RP. Eletromiografia aplicada aos músculos da mastigação. Acta Ortop Bras. 2006; 14(2): 106-7.

23. Oliveira JHP, Filho MM, Lima NS, Moraes KJR, Regis RMF, Silva HJ. Estudo da atividade elétrica dos músculos masseter e temporal em adultos jovens durante a mastigação com diferentes alimentos / Study of electrical activity of masseter and temporal muscles in young adults during chewing with different foods. Neurobiologia. Jul-set, 2010; 73 (3): 87-109.

24. Pasinato F, Corrêa ECR, Souza JÁ. Avaliação fotogramétrica da postura da cabeça e coluna cervical de indivíduos com disfunção temporomandibular. Ter. man. Jan- fev, 2009; 7(29):47-53.

25. Sacco ICN, Alibert S, Queiroz BWC, Pripas D, Kieling I, Kimura AA, et al. Confiabilidade da fotogrametria em relação a goniometria para avaliação postural de membros inferiores. Rev. bras. fisioter. São Carlos, set-out, 2007; 11(5): 411-7.

26. Sanchez HM, Barreto RR, Baraúna MA, Canto RST, Morais EG. Avaliação postural de indivíduos portadores de deficiência visual através da biofotogrametria computadorizado. Fisioter. Mov. Abr-jun, 2008; 21(2):11-20.

27. Iunes DH, Bevilaqua-Grossi D, Oliveira AS, Castro FA, Salgado HS. Análise comparativa entre avaliação postural visual e por fotogrametria computadorizada. Rev Bras Fisioter. Jul-ago, 2009; 13 (4): 308-15.

28. Motoyoshi M, Shimazaki T, Sugai T, Namura S. Biomechanical influences of head posture on occlusion: an experimental study using finite element analysis. Eur J Orthod. Aug, 2002; 24(4):319-26.

29. Shimazaki T, Motoyoshi M, Hosoi K, Namura $\mathrm{S}$. The effect of occlusal alteration and masticatory imbalance on the cervical spine. Eur J Orthod. Oct, 2003; 25(5):457-63.

30. Catanzariti JF, Debuse T, Duquesnoy B. Chronic neck pain and masticatory dysfunction. Joint Bone Spine. 2005; 72: 515-9.

31. Schinestsck PAN, Schinestsck AR. A importância do tratamento precoce da má-oclusäo dentária para o equilíbrio orgânico e postural / The importance of early malocclusion treatment importance to organic and postural balance. J. bras. ortodontia ortop. maxilar. Jan-fev, 1998; 3(13):15-30. 